\title{
Internal and environmental secular evolution of disk galaxies
}

\author{
John Kormendy \\ Department of Astronomy, University of Texas at Austin, Austin, TX 78712, USA; \\ Max-Planck-Institut für Extraterrestrische Physik, Garching bei München, Germany; \\ Universitäts-Sternwarte, Ludwig-Maximilians-Universität, München, Germany; \\ email: kormendy@astro.as.utexas.edu
}

\begin{abstract}
This Special Session is devoted to the secular evolution of disk galaxies. Here 'secular' means 'slow'; i.e., evolution on time scales that are generally much longer than the galaxy crossing or rotation time. Internal and environmentally driven evolution both are covered.

I am indebted to Albert Bosma for reminding me at the 2011 Canary Islands Winter School on Secular Evolution that our subject first appeared in print in a comment made by Ivan King (1977) in his introductory talk at the Yale University meeting on The Evolution of Galaxies and Stellar Populations: 'John Kormendy would like us to consider the possibility that a galaxy can interact with itself... I'm not at all convinced, but John can show you some interesting pictures.' Two of the earliest papers that followed were Kormendy (1979a, b); the first discusses the interaction of galaxy components with each other, and the second studies these phenomena in the context of a morphological survey of barred galaxies. The earliest modeling paper that we still use regularly is Combes \& Sanders (1981), which introduces the now well known idea that box-shaped bulges in edge-on galaxies are side-on, vertically thickened bars.
\end{abstract}

It is gratifying to see how this subject has grown since that time. Hundreds of papers have been written, and the topic features prominently at many meetings (e.g., Block et al. 2004; Falcoń-Barroso \& Knapen 2012, and this Special Session). My talk here introduces both internal and environmental secular evolution; a brief abstract follows. My Canary Islands Winter School review covers both subjects in more detail (Kormendy 2012). Kormendy \& Kennicutt (2004) is a comprehensive review of internal secular evolution, and Kormendy \& Bender (2012) covers environmental evolution. Both of these subject make significant progress at this meeting.

Secular evolution happens because self-gravitating systems evolve toward the most tightly bound configuration that is reachable by the evolution processes that are available to them. They do this by spreading - the inner parts shrink while the outer parts expand. Significant changes happen only if some process efficiently transports energy or angular momentum outward. The consequences are very general: evolution by spreading happens in stars, star clusters, protostellar and protoplanetary disks, black hole accretion disks and galaxy disks. This meeting is about disk galaxies, so the evolution most often involves the redistribution of angular momentum.

We now have a good heuristic understanding of how nonaxisymmetric structures rearrange disk gas into outer rings, inner rings and stuff dumped onto the center. Numerical simulations reproduce observed morphologies very well. Gas that is transported to small radii reaches high densities that are seen in CO observations. Star formation rates measured (e.g.) in the midinfrared show that many barred and oval galaxies grow, on timescales of a few Gyr, dense central 'pseudobulges' that are frequently mistaken for classical (elliptical-galaxy-like) bulges but that were grown slowly out of the disk (not made rapidly by major mergers). Our resulting picture of secular evolution accounts for the richness observed in morphological classification schemes such as those of de Vaucouleurs (1959) and Sandage (1961). State-of-the art morphology discussions include the de Vaucouleurs Atlas of Galaxies (Buta et al. 2007) and Buta (2012, 2013).

Pseudobulges as disk-grown alternatives to merger-built classical bulges are important because they impact many aspects of our understanding of galaxy evolution. For example, they are observed to contain supermassive black holes (BHs), but they do not show the well known, tight correlations between BH mass and host properties (Kormendy et al. 2011). We can distin- 
guish between classical and pseudo bulges because the latter retain a 'memory' of their disky origin. That is, they have one or more characteristics of disks: (1) flatter shapes than those of classical bulges, (2) correspondingly large ratios of ordered to random velocities, (3) small velocity dispersions $\sigma$ with respect to the Faber-Jackson correlation between $\sigma$ and bulge luminosity, (4) spiral structure or nuclear bars in the 'bulge' part of the light profile, (5) nearly exponential brightness profiles and (6) starbursts. None of the above classification criteria are $100 \%$ reliable. Published disagreements on (pseudo)bulge classifications usually result from the use of diffferent criteria. It is very important to use as many classification criteria as possible. When two or more criteria are used, the probability of misclassification becomes very small.

I also review environmental secular evolution - the transformation of gas-rich, star-forming spiral and irregular galaxies into gas-poor, 'red and dead' S0 and spheroidal ('Sph') galaxies. I show that Sph galaxies such as NGC 205 and Draco are not the low-luminosity end of the structural sequence (the 'fundamental plane') of elliptical galaxies. Instead, Sph galaxies have structural parameters like those of low-luminosity S+Im galaxies. Spheroidals are continuous in their structural parameters with the disks of S0 galaxies. They are bulgeless S0s. S+Im $\rightarrow \mathrm{S} 0+\mathrm{Sph}$ transformation involves a variety of internal (supernova-driven baryon ejection) and environmental processes (e.g., ram-pressure gas stripping, harassment, and starvation). Improved evidence for galaxy transformation is presented in several papers at this meeting.

Keywords. galaxies: bulges; galaxies: evolution; galaxies: structure

\section{Acknowledgements}

My work is supported by the Curtis T. Vaughan, Jr. Centennial Chair in Astronomy at the University of Texas at Austin and by the Max-Planck-Institut für Extraterrestrische Physik (MPE). This review was written during a visit to MPE and to the UniversitätsSternwarte of the Ludwig-Maximilians-Universität. I thank MPE Managing Director Ralf Bender and to the staffs of both institutions for their hospitality and support.

\section{References}

Block, D. L., Puerari, I., Freeman, K. C., et al. (eds.) 2004, Penetrating Bars Through Masks of Cosmic Dust: The Hubble Tuning Fork Strikes a New Note (Dordrecht: Kluwer)

Buta, R. 2012, in XXIII Canary Islands Winter School of Astrophysics, Secular Evolution of Galaxies, ed. J. Falcón-Barroso \& J. H. Knapen (Cambridge: Cambridge University Press), in press

Buta, R. 2013, in Planets, Stars, and Stellar Systems, Vol. 6, Extragalactic Astronomy and Cosmology, ed. W. C. Keel (New York: Springer-Verlag), in press (arXiv:1102.0550)

Buta, R. J., Corwin, H. G. \& Odewahn, S. C. 2007, The de Vaucouleurs Atlas of Galaxies (Cambridge: Cambridge University Press)

Combes, F. \& Sanders, R. H. 1981, A\&A A, 96, 164

de Vaucouleurs, G. 1959, Handbuch der Physik, 53, 275

Falcón-Barroso, J. \& Knapen, J. H. (eds.) 2012, XXIII Canary Islands Winter School of Astrophysics, Secular Evolution of Galaxies (Cambridge: Cambridge University Press)

King, I. R. 1977, in The Evolution of Galaxies and Stellar Populations, ed. B. M. Tinsley \& R. B. Larson (New Haven: Yale University Observatory), p. 1

Kormendy, J. 1979a, in Photometry, Kinematics and Dynamics of Galaxies, ed. D. S. Evans (Austin: Department of Astronomy, University of Texas at Austin), p. 341

Kormendy, J. 1979b, ApJ, 227, 714

Kormendy, J. 2012, in XXIII Canary Islands Winter School of Astrophysics, Secular Evolution of Galaxies, ed. J. Falcón-Barroso \& J. H. Knapen (Cambridge: Cambridge Univ. Press), p. 1

Kormendy, J. \& Bender, R. 2012, ApJS, 198, 2

Kormendy, J., Bender, R. \& Cornell, M. E. 2011, Nature, 469, 374

Kormendy, J. \& Kennicutt, R. C. 2004, ARAA, 42, 603

Sandage, A. 1961, The Hubble Atlas of Galaxies (Washington: Carnegie Inst. of Washington) 